\title{
Mean-Semivariance Portfolio Optimization with Multiobjective Evolutionary Algorithms and Technical Analysis Rules
}

\author{
Luís Lobato Macedo ${ }^{1,4, *}$, Pedro Godinho ${ }^{2,4}$, Maria João Alves ${ }^{2,3,4}$ \\ luis.lobato.macedodat.gov.pt, pgodinhodfe.uc.pt, mjalves@fe.uc.pt \\ ${ }^{1}$ Revenue \& Customs, Deputy Directorate-General for Tax Collection, Lisbon, Portugal \\ ${ }^{2}$ CeBER - Center for Business and Economics Research, University of Coimbra, Portugal \\ ${ }^{3}$ INESC, Coimbra, Portugal \\ ${ }^{4}$ Faculty of Economics, University of Coimbra, Coimbra, Portugal \\ * Corresponding author
}

\begin{abstract}
.
Recent work has been devoted to study the use of multiobjective evolutionary algorithms (MOEAs) in stock portfolio optimization, within a common mean-variance framework. This article proposes the use of a more appropriate framework, mean-semivariance framework, which takes into account only adverse return variations instead of overall variations. It also proposes the use and comparison of established Technical Analysis (TA) indicators in pursuing better outcomes within the risk-return relation. Results show there is some difference in the performance of the two selected MOEAs - Non-dominated Sorting Genetic Algorithm II (NSGA II) and Strength Pareto Evolutionary Algorithm 2 (SPEA 2) - within portfolio optimization. In addition, when used with four TA based strategies - Relative Strength Index (RSI), Moving Average Convergence/Divergence (MACD), Contrarian Bollinger Bands (CBB) and Bollinger Bands (BB), the two selected MOEAs achieve solutions with interesting in-sample and out-of-sample outcomes for the BB strategy.
\end{abstract}

Keywords: Multiobjective Optimization; Evolutionary Algorithms; Stock Portfolio; Mean-Semivariance; Technical Analysis

(C) 2017. This manuscript version is made available under the CC-BY-NC-ND 4.0 license http://creativecommons.org/licenses/by-nc-nd/4.0/

This is a post-peer-review, pre-copyedit version of an article published in Expert Systems with Applications. The final version of the article is available online at: http://dx.doi.org/10.1016/j.eswa.2017.02.033 


\section{Introduction}

In recent years, some metaheuristic-based research has been devoted to the study of Finance (Ni \& Wang, 2013). One specific area of interest is portfolio selection, where different types of metaheuristics have been employed in the optimization process (Metaxiotis \& Liagkouras, 2012), both under single objective and multiobjective perspectives. The latter perspective gathered popularity in this particular area, since portfolios focus on two major objectives: return and risk.

Some of the metaheuristics most often applied to portfolio optimization are evolutionary algorithms (EA), both in single objective (Talebi, Molaei, \& Sheikh, 2010) and in multiobjective frameworks (Mishra, Panda, \& Majhi, 2014). These latter studies use multiobjective algorithms to search for solutions as near as possible to the efficient frontier (Pareto front), enhancing results in each iteration by selecting solutions progressively closer to the true Pareto front. The Pareto front is the set of non-dominated solutions of a multiobjective optimization problem, where it is not possible to improve the value of one objective function without simultaneously deteriorating the value of another objective function. However, for simplicity reasons, when we mention the non-dominated solution set, we are referring to the best set of solutions at any given iteration of the multiobjective evolutionary algorithm (MOEA).

The present article intends to present and analyse the results of two different MOEAs - the Nondominated Sorting Genetic Algorithm - NSGA II (Deb, Pratap, Agarwal, \& Meyarivan, 2002) and the Strength Pareto Evolutionary Algorithm - SPEA 2 (Zitzler, Laumanns, \& Thiele, 2001) in the selection of assets (stocks), where the decision to be long in the market or out-of-the-market is determined by the signal generated through the use of a technical analysis (TA) indicator (Fu, Chung, \& Chung, 2013). We will use four different TA trading strategies and develop a comparative analysis. The selected trading strategies are the following: Moving Average Convergence / Divergence (MACD), Relative Strength Index (RSI), Bollinger Bands (BB) and Contrarian Bollinger Bands (CBB).

The main contribution of this work is twofold - first, to compare the performance of two up-to-date MOEAs (NSGA II and SPEA 2) in a mean-semivariance portfolio optimization framework, both in-sample and out-of-sample; second, to assess how the use of trading strategies based on TA indicators may (or may not) lead to improve portfolio performance. We have also conducted a stratified market analysis, studying the behaviour of the optimization process in markets with different degrees of liquidity and efficiency, and assessing how these factors may influence performance.

This paper is structured as follows. After this brief introduction, Section 2 describes the multiobjective portfolio optimization problem as well as the rationale for using semi-variance as a measure of risk. Section 3 presents the two aforementioned MOEAs used in this work. Section 4 introduces the adopted TA trading strategies. The employed methodology is described in section 5. Empirical results are reported and discussed in Section 6. In Section 7 the major conclusions of this study are presented. 


\section{Mean-Semivariance Portfolio Optimization}

Portfolio optimization aims at choosing the proportions of different stocks (or other market assets) to integrate a portfolio with the purpose of maximizing overall expected return and minimizing overall risk. In financial markets, there is a trade-off between return and risk. In general, the better we get in terms of return (greater return), the worse-off we will be regarding risk (larger risk). Different combinations of assets weights (proportions of the amount allocated to each asset with reference to the available financial amount) provide different outputs regarding expected return and risk. The aim of the optimization task is to reach the set of combinations where, for any given level of risk, it is impossible to increase expected return, or, for any given level of expected return, it is impossible to decrease the level of risk. This set of combinations constitutes the Pareto front of the bi-objective optimization problem.

Traditionally, portfolio optimization problems use variance (or standard deviation) as a measure of risk. Although commonly accepted, this measure is not the most appropriate for assessing risk, since it considers equally adverse deviations (below average) as well as favourable ones (above average). However, as Markowitz

admitted, an investor is only concerned with adverse variations. In this context, Markowitz proposed an alternative measure of risk, the "Semivariance" (Markowitz, 1991), which considers only adverse deviations. Semivariance is mathematically defined as

$$
S=E\left(\min \left(0, R_{P}-C\right)^{2}\right)
$$

where $E(\cdot)$ is the expected value, $R_{P}$ is the portfolio return and $C$ is a benchmark. The difficulty with the computation of semivariance resides on the endogenous nature of the portfolio semicovariance matrix, which depends on the weights given to each asset, i.e., a change in weights affects the periods in which the portfolio underperforms the benchmark " $C$ ". Markowitz proposed a problem reformulation (Markowitz, 1993) including additional variables in order to use the Critical Line Algorithm (CLA) as in the classical case of Mean-Variance portfolio optimization. Still, the complexity of the method stands as an inconvenience to this alternative. (Estrada, 2008) presented a simpler way to deal with the endogeneity problem: the author proposed a heuristic approach to obtain a symmetric and exogenous semicovariance matrix, which can be used to approximate portfolio semivariance. Estrada argued that this heuristic yields a portfolio semivariance that is both highly correlated and very close to the actual value it intends to approximate. The method of Estrada allows simplification and reduces the computational effort required for the calculations. However, we must point out that this is still an approximation, introducing inaccuracies to the solutions.

When using MOEAs, the difficulty of computing the semivariance is overcome by nature, since the computation is made by iterations and, in each iteration, the weights of the portfolio(s) are known a priori because they are generated by the algorithm. Knowing the weights, we may compute the portfolio average return (used as the benchmark) and consequently determine in which periods the portfolio underperforms the benchmark. Therefore, in this respect, MOEAs seem to be adequate tools for portfolio optimization under a mean-semivariance framework. 
The multiobjective portfolio optimization problem that we consider can be formulated as follows:

$$
\begin{gathered}
\max E\left(R_{P}\right)=\sum_{i=1}^{n} w_{i} E\left(R_{i}\right)=\sum_{t=1}^{T} \sum_{i=1}^{n} w_{i} \frac{R_{i t}}{T} \\
\min S\left(R_{P}\right)=\sum_{i=1}^{n} \sum_{j=1}^{n} w_{i} w_{j} S_{P C}
\end{gathered}
$$

Subject to:

$$
\begin{gathered}
\sum_{i=1}^{n} w_{i}=1 \\
S_{P C}=E\left(\min \left(0, R_{P}-C\right)^{2}\right)=\frac{1}{T} \sum_{t=1}^{T}\left[\min \left(0, R_{P t}-C\right)\right]^{2}
\end{gathered}
$$

where $P$ stands for portfolio, $w_{i}$ is the weight of asset $i$ in the portfolio, $R_{P}$ is the portfolio overall return, $R_{i t}$ is the return of asset $i$ in period $t, n$ is the number of assets, $S$ is the semivariance (as defined by Markowitz) and $T$ is the number of periods considered in the analysis. In this work, the benchmark is $C=E\left(R_{P}\right)$.

\section{Multiobjective Evolutionary Algorithms Applied to Portfolio Optimization}

Over the last fifteen years, we have observed a substantial development of multiobjective evolutionary algorithms - (Metaxiotis \& Liagkouras, 2012), (Deb, 2001), (Coello, Lamont, \& Veldhuizen, 2007) - and some of these algorithms have reached a high level of acceptance as efficient means to obtain good solutions for complex problems within a reasonable amount of time. To the most popular, attempts to enhance their performance succeeded and, consequently, new versions of the algorithms emerged. That is the case of the NSGA II and the SPEA 2 algorithms.

NSGA II was first presented in (Deb et al., 2002) and it emerged as an improvement to the original NSGA. Several studies have emphasized the good performance of this algorithm when compared with other MOEAs. NSGA II has been found particularly effective by adding a few adjustments (adopting a «predatorprey approach») when dealing with 2 or 3 objectives (Deb, Sundar, Rao N., \& Chaudhuri, 2006). New improvements to this algorithm have been introduced in (D'Souza, Sekaran, \& Kandasamy, 2010), leading to the reduction of its running time and complexity, and therefore making it more attractive for solving practical problems. Another variation of the original NSGA (Michalak, 2016) includes the use of an external population in the optimization process. This variation led to better results, although it was only tested in some benchmark problems associated with specific functions (ZDT-1, ZDT-2, ZDT-3, ZDT-4 and ZDT-6). SPEA 2 (Zitzler et al., 2001) results from an improvement of the first SPEA. In this new algorithm the fitness assignment strategy 
is fine-tuned, considering for each individual solution how many solutions it dominates and is dominated by. SPEA 2 incorporates a nearest neighbour density estimation technique that enhances the optimization process.

Both NSGA II and SPEA 2 have been considered as two of the best currently available MOEAs and they are often used in comparative studies within multiobjective problems, e.g. (Mishra, Panda, Meher, \& Majhi, 2010), (Anagnostopoulos \& Mamanis, 2011), (Lwin, Qu, \& Kendall, 2014). One particular area in which these algorithms have been successfully applied is stock portfolio optimization. Considering two objective functions, risk and return, the problem of portfolio optimization possesses the very characteristics MOEAs are tailored for, especially when taking into consideration some more realistic assumptions, like cardinality (maximum number of admissible stocks in the portfolio), round-lot constraints (stocks/assets often trade in standard units, called the lots, composed by a predetermined number of shares, usually but not necessarily 100 ; see (Skolpadungket, Dahal, \& Harnpornchai, 2007), or the incorporation of trading costs. Such constraints make the use of the CLA very difficult or impracticable. In this kind of problems, both SPEA 2 and NSGA II have been references in the literature, and they usually present consistently better results than alternative techniques - (Duran, Cotta, \& Fernández, 2009), (Mishra, Panda, Meher, \& Sahu, 2009), (Metaxiotis \& Liagkouras, 2012). We will use these algorithms implemented in Matlab Release $2016 a$.

\section{$4 \quad$ Technical Analysis}

TA consists in using price data and occasionally trading volume (number of transactions occurred during a trading session) to anticipate and predict future price movements and exploit them financially by trading according to such expected movements. If an investor uses some TA-based rule translated into a signal (buy, sell, stay or get out of the market) and applies it to his trading strategy, he may profit from market movements by using TA. For instance, if the signal generated by the trading rule associated with a TA indicator indicates that the market is prone to a price climb, the investor will buy now expecting to sell later at a higher price. Therefore, if TA rules are effective, the investor will collect above normal profits, on average, during a long period of time. In our work we have considered the Buy \& Hold (B\&H) strategy as the reference for normal profits.

The use of TA in trading has been viewed with scepticism from the Academic world regarding the attainment of above normal returns. Several studies present strong evidence in favour of TA, either based on indicator analysis, such as (Brock, Lakonishok, \& LeBaron, 1992) and (Pinto, Neves, \& Horta, 2015), or based on chart analysis, like (Lo, Mamaysky, \& Wang, 2000). However, other studies, such as (Allen \& Karjalainen, 1999) and (Neely, 2003), acknowledge little value in TA-based strategies; this is particularly so when some more realistic assumptions - like the existence of transaction costs - are considered (Macedo, Godinho, \& Alves, 2016). In this context, it is important to gather further empirical evidence for or against the validity of TA as an effective tool to exploit market inefficiencies, namely in stock markets. We decided to test the optimization algorithms under four common TA strategies: Moving Average Convergence/Divergence (MACD), Relative 
Strength Index (RSI), Bollinger Bands (BB) and Contrarian Bollinger Bands (CBB) and compare them with a simple B\&H scenario.

The Moving Average Convergence/Divergence (MACD) is an indicator created and originally published by (Appel, 1999). This indicator is a combination of price exponential moving averages (EMA), which generates buying and selling signals through a comparison with a signalling function. In the original configuration, the author used time periods of $(n ; m ; t)=(12 ; 26 ; 9)$, corresponding to the fast price, slow price and MACD signal line EMA's period lengths, respectively.

Fast EMA = exponential moving average of the closing price $(\mathrm{n}=12)$

Slow EMA = exponential moving average of the closing price $(\mathrm{m}=26)$

MACD $=$ Fast EMA - Slow EMA

Signal line $=$ exponential moving average of the MACD $(\mathrm{t}=9)$

We will now explain how the EMA can be computed.

First, a constant $k$ is calculated as:

$$
k=\frac{2}{(w s+1)}
$$

where $w s$ is the length of the moving window of the exponential moving average (the window size). Then, each EMA observation is computed by using the value of the previous one, according to:

$$
E M A_{t}(w s)=\left[\text { Close }_{t}-E M A_{t-1}(w s)\right] \cdot k+E M A_{t-1}(w s)
$$

where Close $_{t}$ is the closing price in period $t$.

The initial EMA is determined as a simple moving average. As an example, see table 1.

\begin{tabular}{|r|r|r|}
\hline Day & Close Price & EMA(4) \\
\hline 1 & 15 & \\
\hline 2 & 16 & \\
\hline 3 & 17 & \\
\hline 4 & 19 & 16.75 \\
\hline 5 & 18 & 17.25 \\
\hline 6 & 13 & 15.55 \\
\hline 7 & 14 & 14.93 \\
\hline 8 & 11 & 13.36 \\
\hline 9 & 12 & 12.81 \\
\hline 10 & 15 & 13.69 \\
\hline 11 & 16 & 14.61 \\
\hline 12 & 18 & 15.97 \\
\hline
\end{tabular}

Table 1. Computation of a hypothetical EMA of window size 4.

We may see that in day 4 the $E M A_{4}(4)$ equals a simple average of the Closing Price of days 1, 2, 3 and 4 . For the calculation of $E M A_{5}(4), k$ will be equal to $2 /(4+1)=0.4$ or $40 \%$, and

$$
E M A_{5}(4)=\left[\text { Close }_{5}-E M A_{4}(4)\right] \cdot k+E M A_{4}(4)=[18-16.75] \cdot 0.4+16.75=17.25
$$


In the following day, EMA will be

$$
E M A_{6}(4)=\left[\text { Close }_{6}-E M A_{5}(4)\right] \cdot k+E M A_{5}(4)=[13-17.25] \cdot 0.4+17.25=15.55
$$

and so forth.

The common technical strategy associated with this indicator states that we should have a long ${ }^{1}$ position when the $M A C D$ value rises above the Signal line and be short ${ }^{2}$ in the market when the opposite situation occurs. In our case, since we are considering stocks, we will be long if MACD>Signal and be out of the market if $M A C D \leq$ Signal. We will not assume short positions.

The Relative Strength Index (RSI) is a momentum oscillator conceived to measure relative overbought/oversold market conditions. (Wilder, 1978) presents the structure and the rationale of the index:

$$
R S I_{t}=1-\frac{1}{1+R S_{t}}
$$

where

$$
R S_{t}=\frac{\sum_{i=1}^{n} U_{t-i+1}}{n} / \frac{\sum_{i=1}^{n} D_{t-i+1}}{n}
$$

and

$\sum_{i=1}^{n} U_{t-i+1}=$ sum of price gains (in absolute value) in the $n$ days previous to $t$;

$\sum_{i=1}^{n} D_{t-i+1}=$ sum of price losses (in absolute value) in the $n$ days previous to $t$.

(Wilder, 1978) used $n=14$.

In a situation where the sum of the gains equals the sum of losses the RSI will be 0.5 or $50 \%$, which represents a temporary equilibrium in market conditions (the power of bulls and bears will be offset). On one extreme, if there is a rising streak in market prices and the sum of $D_{t}$ assumes zero value, $R S_{t}$ will tend to infinity and $R S I_{t}$ to $100 \%$. This means the market is completely overbought and is prone for a reversal. On the other hand, in a continuously falling market, the sum of $U_{t}$, and consequently $R S_{t}$, will tend to zero, generating $R S I_{t}=0 \%$. In this situation, the market is extremely oversold and is likely to go up. Empirical evidence in several major markets, over the years since this index was proposed, suggests the existence of a general $30 \%$ $70 \%$ band where the indicator moves most of the time. These figures have been traditionally accepted as oversold and overbought marks. Usually, the investor will be long on an asset whenever its RSI level has hit the oversold threshold and is on an upward trend, indicating price is on the rise. The investor will discard that asset when the RSI level has hit the overbought mark and is moving downwards. However, the indicator is prone to different interpretations, so other market agents may use it in a different way, such as being long when the RSI is on the rise and over $30 \%$ and being short when decreasing and below the $70 \%$ mark. In our specific case, and since we are dealing with stock portfolios, preventing the usage of short positions, the following rule of trading will be adopted: we will be long in the market if RSI $>30 \%$ and the price is on an upward move (price in the present day is larger than price in the previous day), and we will be out of the market otherwise.

\footnotetext{
${ }^{1}$ A long position is a net buying position in the market.

${ }^{2} \mathrm{~A}$ short position is a net selling position.
} 
Bollinger Bands (BB) are a volatility indicator developed and popularized by John Bollinger (Bollinger, 2001). They are represented by the bands generated from a 20-day price moving average plus and minus 2 standard deviations of price changes over the same 20-day period:

$$
\begin{aligned}
M A_{t} & =\frac{\sum_{i=1}^{20} P_{t-i+1}}{20} \\
U B_{t} & =M A_{t}+2 \sigma_{t} \\
L B_{t} & =M A_{t}-2 \sigma_{t}
\end{aligned}
$$

where $M A_{t}$ is the simple moving average, $U B_{t}$ represents the upper band and $L B_{t}$ the lower of the $B B$, all with reference to day $t$, and $\sigma_{t}$ is the standard deviation of price changes for the period of day $t$ and its previous 19 days.

This indicator can be used in different ways. In this work, we will adopt two different trading strategies associated with Bollinger Bands: conventional (BB) and contrarian (CBB) Bollinger Bands trading strategies. In the conventional case, a long position will be taken in day $t$ if price is simultaneously above $L B$ and below $M A$ (to avoid false triggering) at day $t-1$. If these conditions are not met, we will be out of the market. In the $C B B$ case, we will assume a long position in moment $t$ if price is below $U B$ at moment $t-1$. These are commonly adopted trading strategies in the industry, but other valid strategies could be used. For a more detailed study of these indicators, see for instance (Kirkpatrick \& Dahlquist, 2011) or (Kaufman, 2013).

\section{$5 \quad$ Data and methodology}

This section presents the methodology applied to the development of the study. There is a general perception that financial markets behave differently according to the degree of development. Usually, a developed and liquid market is associated with greater levels of efficiency and lack of trading opportunities. Conversely, a less developed and less liquid market is considered to be more prone to exploitable trading opportunities, because agents are not so active checking market inefficiencies. In this work we considered it important to compare trading performance according to the level of perceived efficiency. We have aggregated stocks of countries with economic similarities, according to similar market dimension and perceived efficiency and liquidity, from the least efficient and liquid to the most efficient and liquid, to form 4 different markets: market Tier 1 - stocks of countries in development (Argentina, Brazil and South Africa), market Tier 2 - stocks of peripheral developed countries (Greece, Portugal and Belgium), market Tier 3 - stocks of fully developed countries (UK, Australia and The Netherlands), and finally, stocks of the US market. With these aggregations, we will try to grasp conclusions on the existence of any differences in the different markets with respect to exploitable trading opportunities.

We used daily adjusted closing price data (in EUR) of stocks from Argentina (15), Brazil (17), South Africa (13), Greece (15), Portugal (15), Belgium (15), UK (15), Australia (15), The Netherlands (15) and the US (49), aggregated in 4 different markets. Stock prices span a period of almost 16 years $(15$ 3/4 years, from 
2000-01-03 to 2015-10-01) for all stocks, which are presented in the annex to this article (Tables A1 to A4). Stock price data was extracted from Thompson-Reuters Datastream (now Eikon). Corporations were selected by order of appearance in Datastream queries and according to the required condition of data availability (with data from year 2000 on). The complete price matrices have dimension $T^{*} n$, where $T$ is the total number of price observations and $n$ the number of assets, equal to $4109 * 49$ for the US market and $4109 * 45$ for all other market aggregations (Tiers 1, 2 and 3). In order to evaluate the return of the assets and consequently the portfolios, we adopted the model of continuous compound rates, formulated in (13):

$$
P_{i t}=P_{i(t-1)} \cdot \exp ^{R_{i t}}
$$

where

$P_{i t}$ is the price of asset $i$ at day $t$;

$R_{i t}$ is the rate of return of asset $i$ at day $t$.

The rate of return at day $t$, for any asset $i$, is determined by (14):

$$
R_{i t}=\ln \left(P_{i t}\right)-\ln \left(P_{i(t-1)}\right)
$$

That is, as we are dealing with daily price observations, the continuously compounded daily rate of return of an asset is equal to the difference between the natural logarithm of its close price at one day and the natural logarithm of its close price of the previous day. Applying expression (14) to the matrix of prices we obtain a matrix of daily returns, from 2000-01-04 to 2015-10-01 (dimension $(T-1) * n$ ). To each matrix of daily returns, a column of 4108 observations is added, with a daily return of 0.0000766551 , corresponding to an annual risk free rate of return of 0.02 for the Cash asset. Therefore, the full return matrices have dimension $4108 * 50$ for the US market and $4108^{*} 46$ for all the other aggregate markets (Tiers 1, 2 and 3), and represent the B\&H scenario.

The matrices under $C B B, B B, M A C D$ and $R S I$ strategies are obtained by applying the rules mentioned in section 4. These rules produce four new matrices for each market, one for each of the four TA trading strategies, where each element assumes value 1 or 0 depending on the outcome of the respective rules (" 1 " for a long position; " 0 " for out of the market). These new binary matrices have the same dimension of the previous B\&H return matrix. Subsequently, we proceed to an element-wise multiplication of the binary matrix with the original $\mathrm{B} \& \mathrm{H}$ matrix, creating $C B B, B B, M A C D$ and $R S I$ return matrices, with daily returns (identical to the $\mathrm{B} \& \mathrm{H}$ return) for the assets (except Cash) when the rule implies being long in the market and zero returns for the days when the trading signal implies being out of the market.

These 20 return matrices $(\mathrm{B} \& \mathrm{H}, \mathrm{CBB}, \mathrm{BB}, \mathrm{MACD}$ and RSI strategies times the 4 considered markets US market and market aggregations designated by Tier 1, Tier 2 and Tier 3) all include trading costs, i.e., daily returns are deducted of their respective trading costs. Nowadays, with the advent of internet based trading apps 
and brokerage firms' fierce competition, trading costs may assume infinitesimal percentages ${ }^{3}$. For the purpose of this work, we consider a percentage trading cost over trading amounts of $0.05 \%$ per round-turn. A roundturn is a complete cycle of buying and selling an asset, so that for every round-turn there are two trades (one buy and one sell). Each of the return matrices is then split into 2 different matrices, one for in-sample data (first 10 years, 2000-01-04 to 2009-12-31) and another for out-of-sample data (last 5 3/4 years, from 2010-01-02 to 2015-10-01).

In the optimization process using NSGA II and SPEA 2, the genes that form a single chromosome represent the asset weights in the portfolio. A chromosome is therefore a vector $\left(w_{1}, w_{2}, \ldots, w_{n}\right)$, with $w_{i} \in \mathbb{R}$, $w_{i} \geq 0, i=1,2, \ldots, n$, and $\sum_{i=1}^{n} w_{i}=1 ; n=46$ in Markets Tier 1 to 3 and $n=50$ in the US market. Each chromosome represents a solution (portfolio). The sum of all weights $w_{i}, i=1, \ldots, n$ is normalized to 1 after crossover and mutation and before fitness evaluation, i.e. $w_{i}=w_{i}^{\prime} / W,=1, \ldots, n, W=\sum_{i=1}^{n} w_{i}^{\prime}$, where $w_{i}^{\prime}$ represents the weight of the $i^{\text {th }}$ asset after the algorithm operations and $w_{i}$ is the normalized weight.

When combining the weights determined by the algorithm with the trading signal generated by TA indicators, some assets may become inactive, i.e., out of the market. When an asset is inactive, its daily return is null in the return matrix, making its weight void. For instance, suppose a portfolio is composed by 5 different assets, each with an equal weight of $20 \%$. At some moment, due to the application of TA rules, one of the assets is out of the portfolio. The 4 remaining stocks have the same equal relevance in that portfolio, i.e., their proportion is $20 \%$ in an aggregate of $80 \%$, which means that each active asset represents $25 \%=20 / 80$ of the invested capital. For every iteration, the weight values (genes) in each portfolio (chromosome) represent the relative importance of their assets. When all assets are active in the portfolio, such as in the case of the B\&H strategy, the weights represent the percentage of each asset in the portfolio. Whenever any asset is out of the market, as a result of the applied TA strategy, its weight should be divided by the sum of the weights of all active assets in order to interpret the real importance of each active asset.

We have considered the following parameterizations for the algorithms NSGA II and SPEA 2:

- Initial population: $n_{\text {pop }}=100$ chromosomes;

- Maximum number of elements in the archive of non-dominated solutions: $n_{\text {arch }}=$ 100 chromosomes; this archive is the outcome of the algorithm (the optimized solutions)

- Maximum number of iterations: $n_{\text {iter }}=300$

- Crossover probability: $p_{c}=0.9$

- Mutation probability: $p_{m}=0.02$

The experiment setting follows the steps below.

1. For all markets (Tier 1, 2, 3 and US) and strategies (B\&H, CBB, BB, MACD and RSI), do:

\footnotetext{
${ }^{3}$ See for instance Robinhood Brokerage Services, a company created by two Stanford University graduates that offers zero commissions for the use of their online app https://www.robinhood.com/.
} 
a. Optimize populations of 100 randomly generated solutions by applying the multiobjective algorithm (NSGA II / SPEA 2) to in-sample (IS) data; perform 25 independent runs, each run producing a population of at most 100 non-dominated solutions (portfolios);

b. Compute the hypervolume (HV) measure (Zitzler \& Thiele, 1999) for the populations of nondominated solutions and select the population with median $\mathrm{HV}$ value among the 25 independent runs;

c. Use the weights of the solutions in the selected population and apply them to out-of-sample (OOS) data in order to generate the respective OOS population of solutions;

2. Compare the optimized populations (one per market) for the 5 strategies, for both the IS and the OOS data.

The result of the optimization process using each MOEA in the IS data is a sample of 25 sets (frontiers) of at most 100 optimized solutions per each combination market-strategy. In order to compare frontiers it would be unpractical to use all samples, especially when using graphical representations. Therefore, we decided to use a criterion to select a representative frontier in each combination. The criterion adopted is to rank the 25 frontiers according to their hypervolume (HV) values (Zitzler \& Thiele, 1999) and then selecting the frontier with the median HV value as representative of the MOEA optimization outcome. HV measures the volume of the multidimensional region that is dominated by the set of non-dominated solutions that is being assessed. This quality indicator can assess both convergence and diversity of the non-dominated solutions, and larger values of HV indicate better approximation sets. The code of (Fonseca, Paquete, \& López-Ibáñez, 2006) was used in this study to compute the HV values.

\section{Experimental Results and Discussion}

The main goals of the empirical work are to assess:

1) which of the algorithms presents a better performance both in and out-of-sample (IS vs. OOS);

2) what is the impact of the chosen TA strategies on the non-dominated frontiers;

Figures 1 and 5 present the IS and OOS outcomes (risk-return frontiers of non-dominated portfolios with median HV value), respectively, for all five strategies and for each market, resulting from the application of the NSGA II algorithm. Figures 2 and 6 show the IS and OOS outcomes resulting from the SPEA 2 algorithm. In-sample frontiers result from the optimization process itself; out-of-sample results depict in-sample solutions applied to out-of-sample data.

Regarding the in-sample results obtained with NSGA II (Figure 1), the US market is the one generating the most similar non-dominated frontiers among all strategies, and they are very close to the B\&H median frontier, suggesting a great level of efficiency in this market. The strategy using BB trading rules presents the best IS median non-dominated frontiers for the markets Tier 2 and 3. Conversely, CBB is the strategy that 
produces the median frontiers closest to the B\&H scenario, suggesting little advantage in using its associated trading strategy.

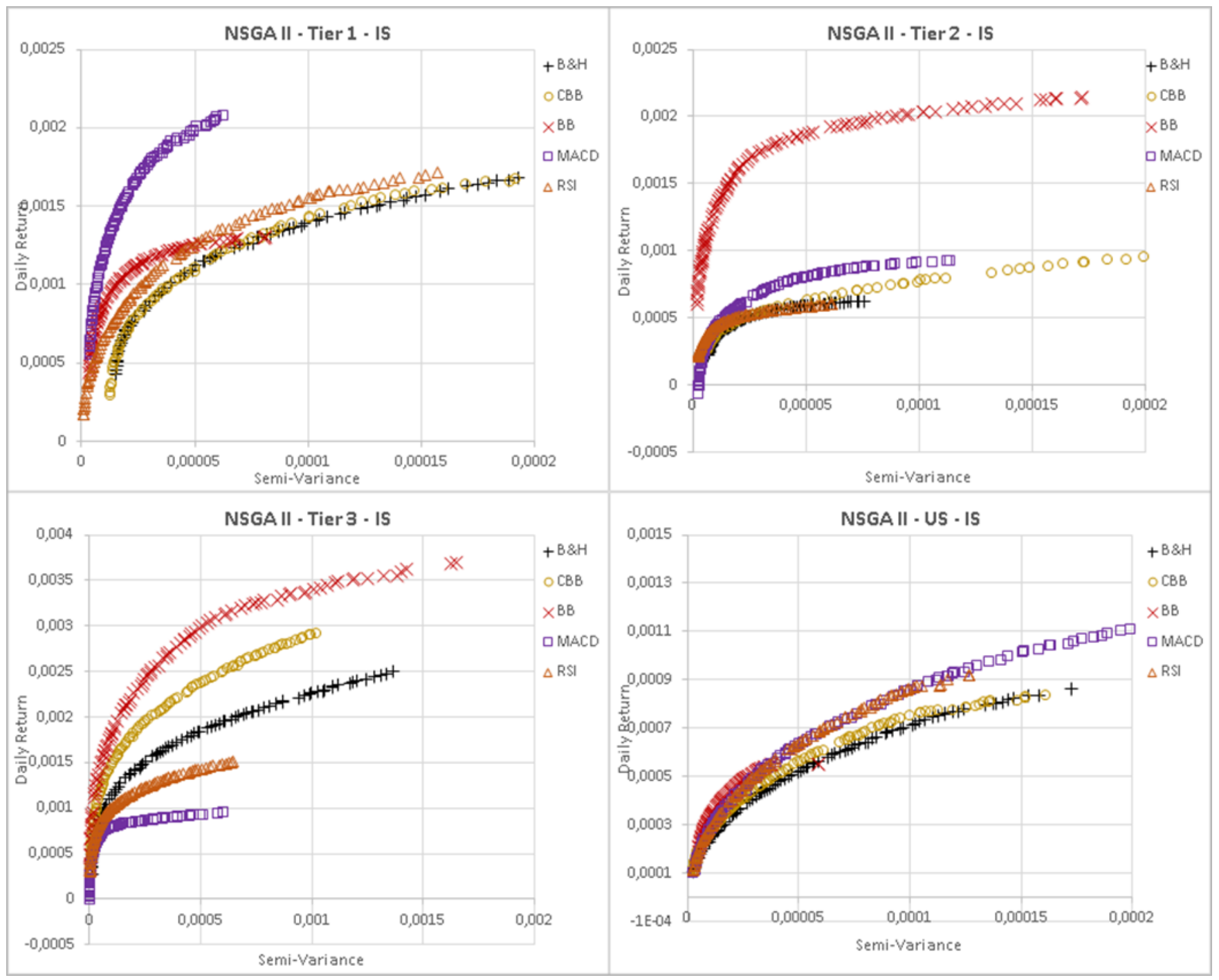

Figure 1 - NSGA II frontiers of non-dominated solutions, in-sample, under 5 strategies (B\&H, CBB, BB, MACD and RSI), with costs, regarding all 4 aggregate markets (Tiers 1, 2, 3 and US).

The market presenting better BB IS results is the Tier 3 , which in theory would be the $2^{\text {nd }}$ most efficient market (following the US stock market). This is interesting since it would be expected that the less efficient the market, the more likely it would be to present exploitable trading opportunities and, therefore, the better the non-dominated frontiers that would result from the optimization process with IS data. This might suggest trading opportunities may not arise in less efficient markets as it was supposed to, and/or market liquidity plays an important role in materialising profit opportunities. Tier 3 also shows how the use of some trading strategies (MACD and RSI) may hurt the overall output, presenting results that are even worse than the B\&H scenario. 
However, we must stress that IS results may be the result of some overfitting, so we must be careful in not giving too much importance to the conclusions regarding the IS performance of the strategies.

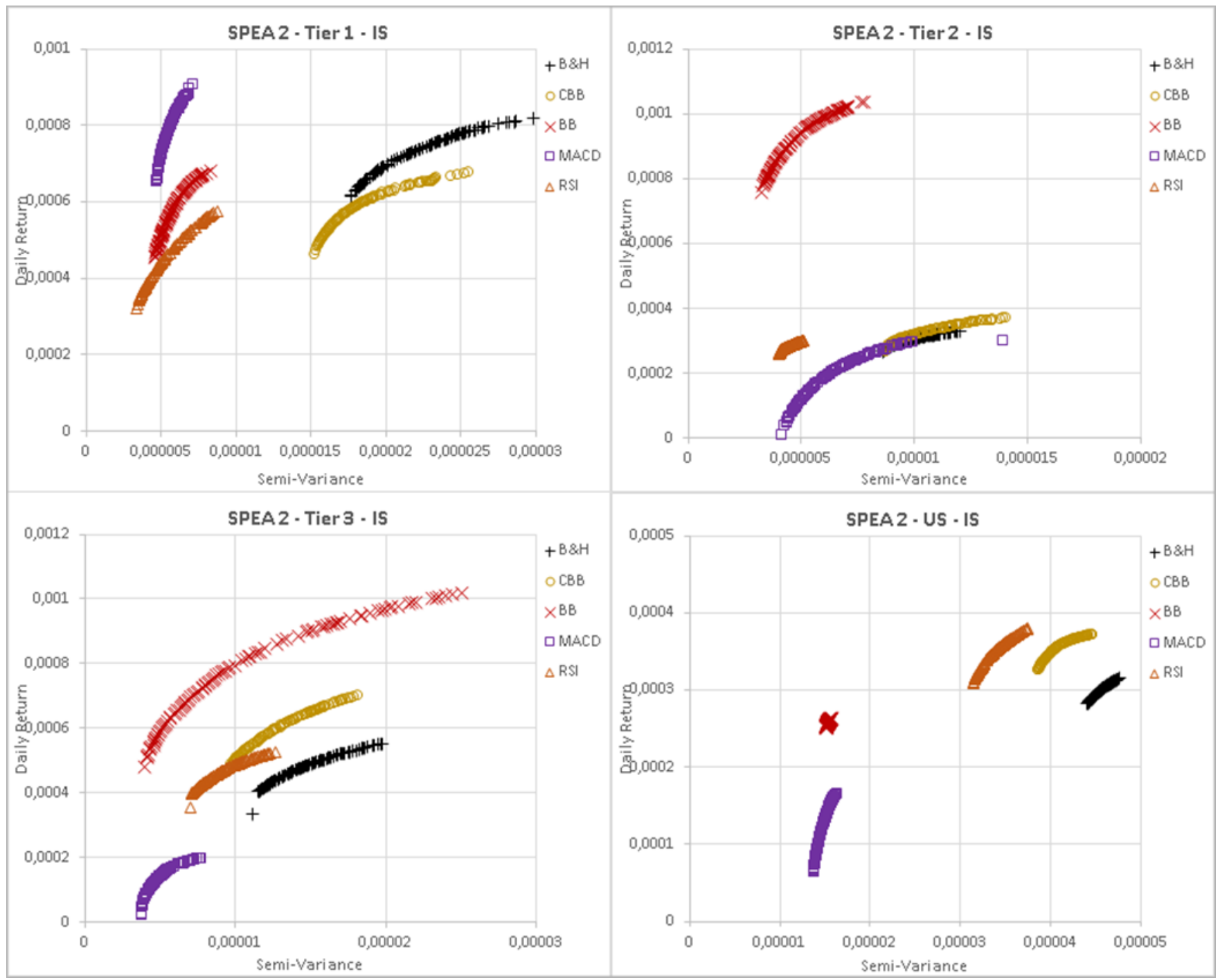

Figure 2 - SPEA 2 frontiers of non-dominated solutions, in-sample, under 5 strategies (B\&H, CBB, BB, MACD and RSI), with costs, regarding all 4 aggregate markets (Tiers 1, 2, 3 and US).

The IS results of SPEA 2 (Figure 2) show a different spectre: the solutions are much more concentrated within a small semi-variance interval, which prevents the possibility of deducing sustained conclusions. Still, we may see BB presents the best median front in markets Tier 2 and 3. The limited extension of the nondominated sets does not allow much further comparison within each market. 


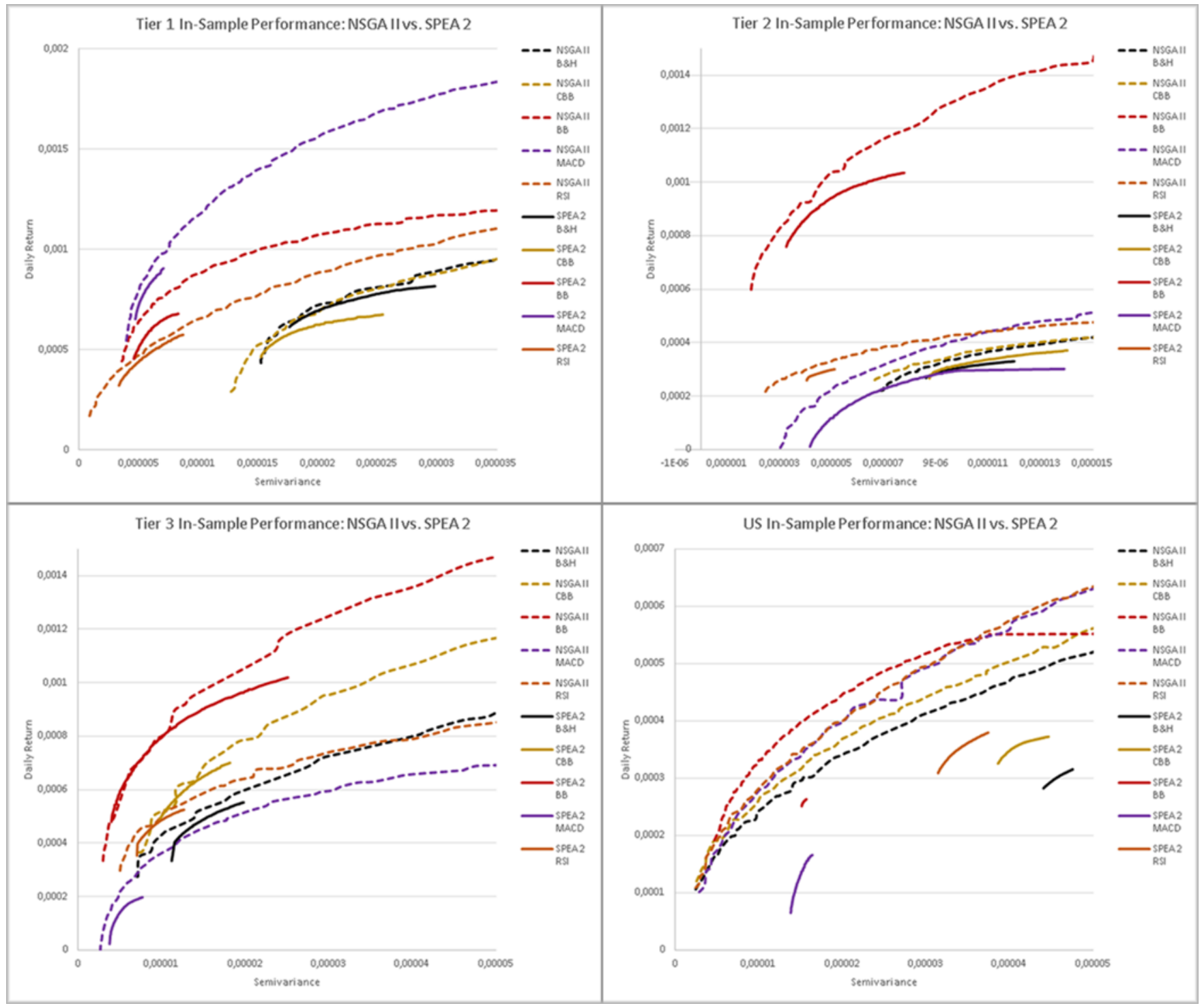

Figure 3 - Performance comparison between NSGA II and SPEA 2 algorithms for the five TA strategies (B\&H, CBB, BB, MACD and RSI) in each market, within the four stock markets (Tiers 1, 2, 3 and US).

The very limited extension of the IS fronts obtained by SPEA 2 is reinforced in Figure 3, which presents a comparison of NSGA II and SPEA 2 performances in IS data. Notice that the scales of semi-variance have been reduced with respect to Figure 1 in order to allow legibility of SPEA 2 fronts; otherwise, it would be almost impossible to see their shapes. The dashed lines represent the NSGA II frontiers for each strategy; the continuous lines stand for the SPEA 2 frontiers. We can observe that in markets Tier 1 to 3 the non-dominated frontiers obtained by both algorithms are very close to each other, but NSGA II consistently outperforms SPEA 2 , for all strategies.

The main conclusions obtained with this comparison (NSGA II outperforming SPEA 2 and SPEA 2 frontiers presenting a lesser extent than those of the NSGA II) are similar to the conclusions reached by (Mishra et al., 2009), (Diosan, 2005) or (Lwin, Qu, \& Zheng, 2013) in portfolio optimization problems. In order to have 
a better perception of this fact, we have further analysed the evolution of the population throughout generations using NSGA II and SPEA 2 in a run for the conventional Bollinger Bands (BB) strategy in the US market (the case that has shown the greatest divergence in terms of front length). Populations in iterations 10, 50, 100, 200 and 300 of the optimization process are presented in Figure 4, so a notion of the population evolution may be inferred. It can be observed that SPEA 2 population starts with a relatively dispersed population but, as it approaches the later iterations, it tends to maintain the front extension or to narrow it slightly, concentrating the population in the middle of the Pareto front. On the other hand, NSGA II stimulates diversity in the population at the same time it approaches the Pareto front.

A possible reason for this discrepancy between the two algorithms may be the way diversity is promoted in the selection procedure of each algorithm. NSGA II uses a crowding distance, which measures the distance of each individual to its nearest neighbours on the objective function space. A non-dominated solution with a smaller value of the crowding distance is more crowded by other solutions and it will be less preferred to integrate next population than another non-dominated solution with higher crowding distance. SPEA 2 uses a density estimation technique. Density information is incorporated in the fitness function to discriminate between individuals having identical raw fitness. The way the density information is calculated and considered in the selection procedure is different from the crowding distance technique of NSGA II. The latter algorithm emphasizes the boundary solutions of the non-dominated set, finding solutions closer to the outlying edges of the Pareto front. This fact may lead NSGA II to provide a broader range of solutions.

This difference in behaviour is not fully replicated in all combinations market / trading strategy. It seems that some markets and/or strategies are more prone to induce this divergence in the performance of the algorithms, leading us to conclude that data also plays an active relevant role in this matter.

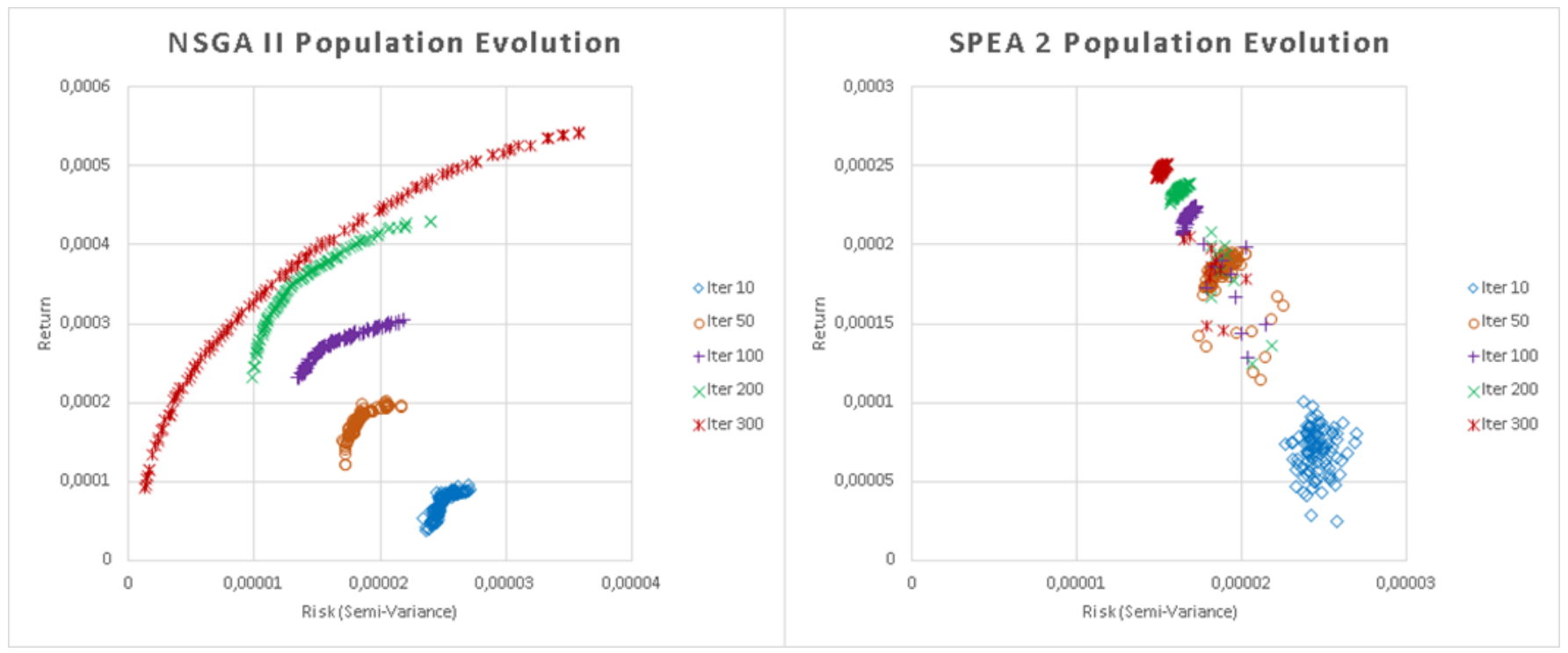

Figure 4 - Comparison of the evolution of NSGA II and SPEA 2 populations throughout iterations for the BB strategy in US market.

Regarding OOS (Figures 5 and 6), overall results obtained by the BB strategy are very interesting. Considering the application of NSGA II a, the BB strategy emerges as the most consistent and giving better 
OOS results among all the TA strategies that were considered. This conclusion confirms the IS results, which in turn suggests the good IS results were not exclusively due to potential overfitting. MACD also reveals some interesting results in OOS data, performing better than B\&H in most markets. The RSI indicator shows better performances in less developed markets. The CBB strategy shows mixed results, with better performances in the US market and Tier 3, and staying close to B\&H in less developed markets (Tiers 1 and 2).

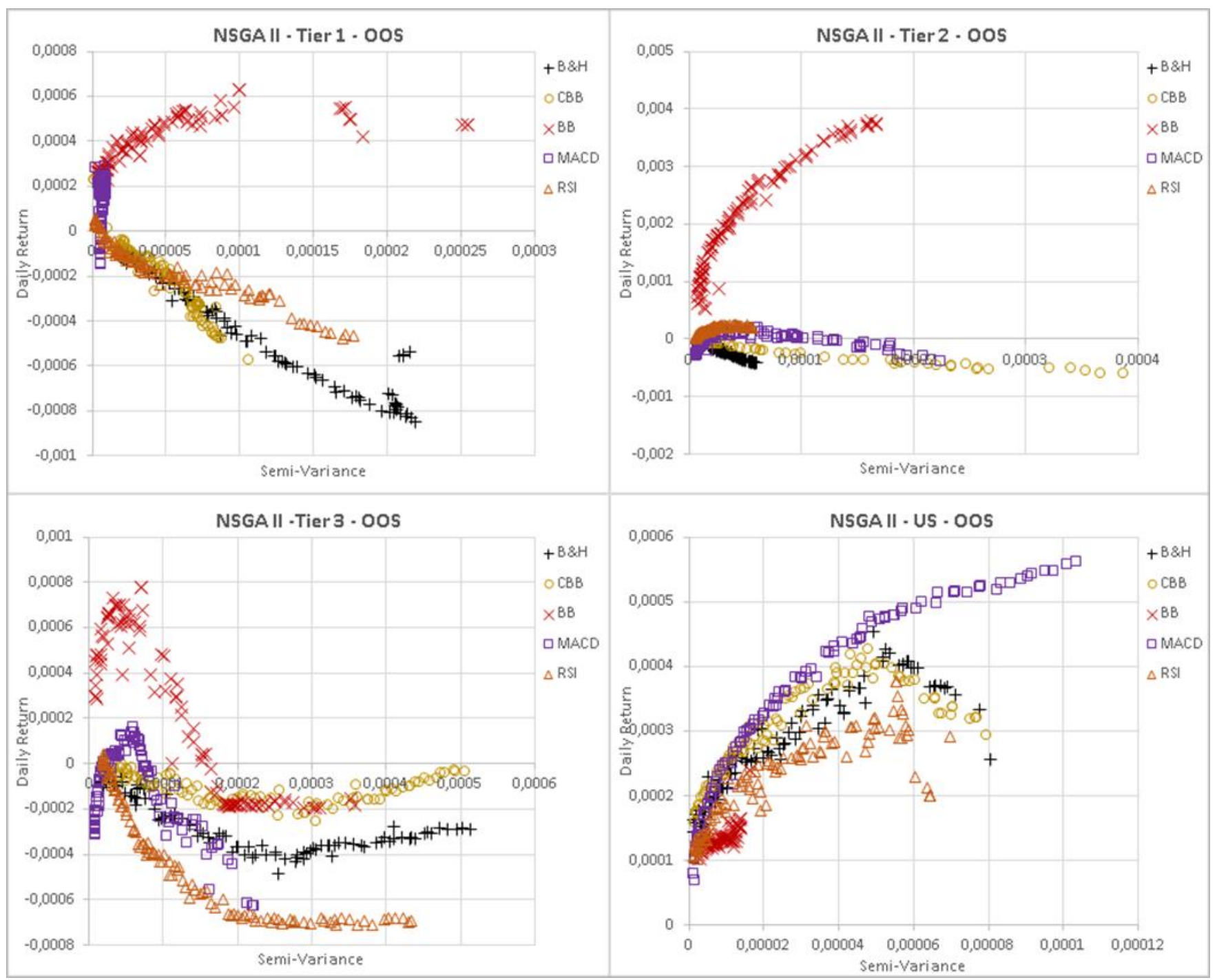

Figure 5 - NSGA II out-of-sample frontiers corresponding to the in-sample non-dominated solutions, under 5 strategies (B\&H, CBB, BB, MACD and RSI), with costs, regarding all 4 aggregate markets (Tiers 1, 2, 3 and US). 


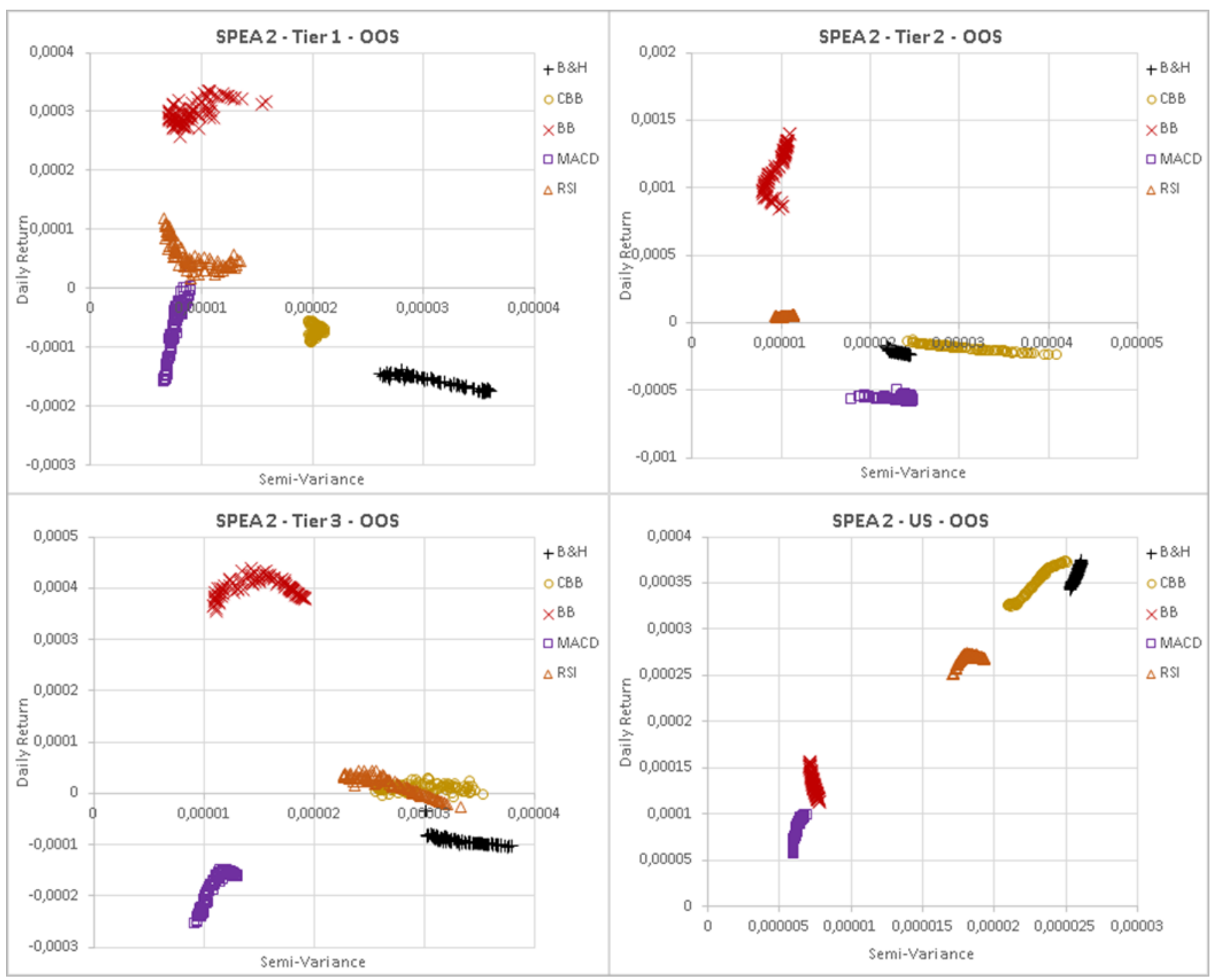

Figure 6 - SPEA 2 out-of-sample frontiers corresponding to the in-sample non-dominated solutions, under 5 strategies (B\&H, CBB, BB, MACD and RSI), with costs, regarding all 4 aggregate markets (Tiers 1, 2, 3 and US).

Similarly to the IS behaviour, OOS results are very limited for solutions generated by SPEA 2. The analysis of SPEA 2 OOS results (figure 6) shows that the BB trading strategy produces the best outcome among all strategies, supporting this trading strategy as the best one within the studied TA techniques. In addition, the results of SPEA 2 suggest that the RSI strategy performs OOS better than B\&H in all markets. The MACD may be considered the strategy with the less interesting results of all, particularly in markets Tier 2 and 3.

\section{Conclusion}

The present work compares the performance of two established multiobjective evolutionary algorithms, NSGA II and SPEA 2, combined with TA trading strategies for mean-semivariance portfolio 
optimization. Five different strategies were used - Buy \& Hold, Contrarian Bollinger Bands, conventional Bollinger Bands, MACD and RSI, all of them incorporating trading costs, for four different markets and two different periods: in-sample, 2000-01-03 to 2009-12-31, and out-of-sample, 2010-01-03 to 2015-10-01. The purpose was twofold: to assess which of the algorithms performed better and to know if the chosen TA indicators influenced the results.

Overall results show that there is quite a difference between the results obtained with NSGA II and with SPEA 2. The former algorithm produces much wider IS non-dominated frontiers, which have similar shapes. On the other hand, the latter algorithm presents IS non-dominated frontiers with different shapes and positions and the solutions are more concentrated presenting low diversity. NSGA II systematically outperforms SPEA 2, in an in-sample comparison. Results also show how the use of TA indicators with associated trading strategies may influence the frontiers of non-dominated portfolios. In most markets, conventional Bollinger Bands presents better out-of-sample results, deeming it as the most profitable TA trading tool and the strategy of election among all studied. The results presented in this article may stimulate further research in the area of portfolio optimization, both using MOEAs and other TA indicators.

\section{References}

Allen, F., \& Karjalainen, R. (1999). Using genetic algorithms to find technical trading rules. Journal of Financial Economics, 51, 245-271.

Anagnostopoulos, K. P., \& Mamanis, G. (2011). The mean-variance cardinality constrained portfolio optimization problem: An experimental evaluation of five multiobjective evolutionary algorithms. Expert Systems with Applications, 38(11), 14208-14217. http://doi.org/10.1016/j.eswa.2011.04.233

Appel, G. (1999). Technical Analysis Power Tools for Active Investors. Financial Times Prentice Hall.

Bollinger, J. A. (2001). Bollinger on Bollinger Bands (1st ed.). McGraw-Hill Education.

Brock, W., Lakonishok, J., \& LeBaron, B. (1992). Simple Technical Trading Rules and the Stochastic Properties of Stock Returns. Journal of Finance, 47(5), 1731-1764.

Coello, C. A. C., Lamont, G. B., \& Veldhuizen, D. A. Van. (2007). Evolutionary Algorithms for Solving Multi-Objective Problems (Second Ed.). Springer-Verlag.

D’Souza, R. G. L., Sekaran, K. C., \& Kandasamy, A. (2010). Improved NSGA-II Based on a Novel Ranking Scheme. Journal of Computing, 2(2), 91-95. Retrieved from http://arxiv.org/abs/1002.4005

Deb, K. (2001). Multi-Objective Optimization Using Evolutionary Algorithms. Chichester, U.K.: Wiley.

Deb, K., Pratap, A., Agarwal, S., \& Meyarivan, T. (2002). A fast and elitist multiobjective genetic algorithm: NSGA-II. IEEE Transactions on Evolutionary Computation, 6(2), 182-197. http://doi.org/10.1109/4235.996017

Deb, K., Sundar, J., Rao N., U. B., \& Chaudhuri, S. (2006). Reference Point Based Multi-Objective 
Optimization Using Evolutionary Algorithms. In International Journal of Computational Intelligence Research (Vol. 2, pp. 273-286). Springer-Verlag. http://doi.org/10.1145/1143997.1144112

Diosan, L. (2005). A multi-objective evolutionary approach to the portfolio optimization problem. In Proceedings of the International Conference on Computational Intelligence for Modelling, Control and Automation (CIMCA-IAWTIC'05). IEEE - Computer Society.

Duran, F. C., Cotta, C., \& Fernández, A. J. (2009). Evolutionary Optimization for Multiobjective Portfolio Selection under Markowitz's Model with Application to the Caracas Stock Exchange. In NatureInspired Algorithms for Optimisation (Vol. 193, pp. 489-509). http://doi.org/10.1007/978-3-642-00267$0 \_18$

Estrada, J. (2008). Mean-Semivariance Optimization: A Heuristic Approach. Journal of Applied Finance, (Spring/Summer), 57-72. Retrieved from http://www.efmaefm.org/0efmameetings/efma annual meetings/2008-athens/estrada.pdf

Fonseca, C. M., Paquete, L., \& López-Ibáñez, M. (2006). An Improved Dimension-Sweep Algorithm for the Hypervolume Indicator. In IEEE Congress on Evolutionary Computation (pp. 3973-3979). Vancouver, BC, Canada: IEEE.

Fu, T. C., Chung, C. P., \& Chung, F. L. (2013). Adopting genetic algorithms for technical analysis and portfolio management. Computers and Mathematics with Applications, 66(10), 1743-1757. http://doi.org/10.1016/j.camwa.2013.08.012

Kaufman, P. (2013). Trading Systems and Methods. Wiley Finance Editions (5th.). John Wiley \& Sons.

Kirkpatrick, C. D., \& Dahlquist, J. R. (2011). Technical Analysis - The Complete Resource for Financial Market Technicians (2nd ed.). FT Press.

Lo, A. W., Mamaysky, H., \& Wang, J. (2000). Foundations of Technical Analysis: Computational Algorithms, Statistical Inference, and Empirical Implementation. The Journal of Finance, 55(4), 17051765.

Lwin, K., Qu, R., \& Kendall, G. (2014). A learning-guided multi-objective evolutionary algorithm for constrained portfolio optimization. Applied Soft Computing, 24, 757-772. http://doi.org/10.1016/j.asoc.2014.08.026

Lwin, K., Qu, R., \& Zheng, J. (2013). Multi-objective Scatter Search with External Archive for Portfolio Optimization. In Proceedings of the 5th International Joint Conference on Computational Intelligence (pp. 111-119). Vilamoura, Algarve, Portugal.

Macedo, L. L., Godinho, P., \& Alves, M. J. (2016). A Comparative Study of Technical Trading Strategies Using a Genetic Algorithm. Computational Economics. http://doi.org/10.1007/s10614-016-9641-9

Markowitz, H. M. (1991). Foundations of Portfolio Theory. Journal of Finance, XLVI(2), 469-477.

Markowitz, H. M. (1993). Computation of mean-semivariance efficient sets by the Critical Line Algorithm. Annals of Operations Research, 45, 307-317. 
Metaxiotis, K., \& Liagkouras, K. (2012). Multiobjective Evolutionary Algorithms for Portfolio Management: A comprehensive literature review. Expert Systems with Applications, 39(14), 11685-11698. http://doi.org/10.1016/j.eswa.2012.04.053

Michalak, K. (2016). Improving the NSGA-II Performance with an External Population. In Intelligent Data Engineering and Automated Learning - IDEAL 2015 (Lecture No, Vol. 9375, pp. 273-280).

Mishra, S. K., Panda, G., \& Majhi, R. (2014). A comparative performance assessment of a set of multiobjective algorithms for constrained portfolio assets selection. Swarm and Evolutionary Computation, 16, 38-51. http://doi.org/10.1016/j.swevo.2014.01.001

Mishra, S. K., Panda, G., Meher, S., \& Majhi, R. (2010). Application of Multi-Objective Evolutionary Algorithms in Computational Finance. In NIT Warangal International Conference. Retrieved from http://dspace.nitrkl.ac.in/dspace/bitstream/2080/1333/1/Application of Multi.pdf

Mishra, S. K., Panda, G., Meher, S., \& Sahu, S. S. (2009). Optimal Weighting of Assets using a Multiobjective Evolutionary Algorithm. International Journal of Recent Trends in Engineering, 2(5), 161166.

Neely, C. J. (2003). Risk-adjusted, ex ante, optimal technical trading rules in equity markets. International Review of Economics and Finance, 12, 69-87.

Ni, H., \& Wang, Y. (2013). Stock index tracking by Pareto efficient genetic algorithm. Applied Soft Computing Journal, 13(12), 4519-4535. http://doi.org/10.1016/j.asoc.2013.08.012

Pinto, J. M., Neves, R. F., \& Horta, N. (2015). Boosting Trading Strategies performance using VIX indicator together with a dual-objective Evolutionary Computation optimizer. Expert Systems with Applications, 42(19), 6699-6716. http://doi.org/10.1016/j.eswa.2015.04.056

Skolpadungket, P., Dahal, K., \& Harnpornchai, N. (2007). Portfolio optimization using multi-obj ective genetic algorithms. In 2007 IEEE Congress on Evolutionary Computation (pp. 516-523). http://doi.org/10.1109/CEC.2007.4424514

Talebi, A., Molaei, M. A., \& Sheikh, M. J. (2010). Performance investigation and comparison of two evolutionary algorithms in portfolio optimization: Genetic and particle swarm optimization. Information and Financial Engineering ICIFE 2010 2nd IEEE International Conference on, 430-437. http://doi.org/10.1109/ICIFE.2010.5609394

Wilder, J. W. (1978). New Concepts in Technical Trading Systems. Trend Research.

Zitzler, E., Laumanns, M., \& Thiele, L. (2001). SPEA2: Improving the Strength Pareto Evolutionary Algorithm (TIK-Report No. 103). TIK-Report.

Zitzler, E., \& Thiele, L. (1999). Multiobjective Evolutionary Algorithms: A Comparative Case Study and the Strength Pareto Approach. IEEE Transactions on Evolutionary Computation, 3(4), 257-271. http://doi.org/10.1109/4235.797969 
ANNEX 


\begin{tabular}{|c|c|c|}
\hline \multicolumn{3}{|c|}{ Market Tier 1} \\
\hline Argentina & Brazil & South Africa \\
\hline AGROMETAL & AMAZONIA ON & ADVTECH \\
\hline BANCO SANTANDER RIO "B" & AMPLA ENERGIA E SERVICOS ON & AFRICAN OXYGEN \\
\hline CAPEX & ATOM PARTICIPACOES & ANGLOGOLD ASHANTI \\
\hline CAPUTO & B MERC BRASIL PN & ARCELORMITTAL SA. \\
\hline CARLOS CASADO "B" & BANCO DO NORD ON & CAXTON \& CTP PB\&PRT. \\
\hline CELULOSA & BANCO ESTADO ESP. SANTO BANEST ON & DATATEC \\
\hline COLORIN & BIC MONARK ON & FIRSTRAND \\
\hline ENDESA COSTANERA & BNCO ALFA INVEST PN & GOLD FIELDS \\
\hline GOFFRE & BOMBRIL PN & HARMONY GOLD MNG. \\
\hline IRSA & CEMEPE INVEST PN & MMI HOLDINGS \\
\hline MORIXE HERMANOS & CIA TECIDOS SANTANENSE PN & NEDBANK GROUP \\
\hline NUEVO BANCO SUQUIA "B" & CNCO.ALFA DE ADMO. SR.F PN & RMB \\
\hline SNIAFA COMR.FINCA. INMB. "A" & CONST AD LIN PN & TSOGO SUN \\
\hline SOCIEDAD COMERCIAL DEL PLATA & CORREA RIBEIRO PN & \\
\hline \multirow[t]{3}{*}{ TRANSPORTADORA DE GAS DEL SUR } & DHB INDUSTRIA E COMERCIO ON & \\
\hline & OI PN & \\
\hline & REDE ENERGIA ON & \\
\hline
\end{tabular}

Table A1 - List of Market Tier 1 stocks used in the portfolio optimization problem.

\begin{tabular}{|c|c|c|}
\hline \multicolumn{3}{|c|}{ Market Tier 2} \\
\hline Greece & Portugal & Belgium \\
\hline AEGEK & CIMENTOS DE PORTL.SGPS & BEFIMMO \\
\hline AEOLIAN INVESTMENT FUND & CIPAN LIMITED DATA & COFINIMMO \\
\hline ALTEC HOLDINGS & COMPTA & COLRUYT \\
\hline ATHENA & COPAM LIMITED DATA & DEXIA \\
\hline ATTICA HOLDINGS & EDP ENERGIAS DE PORTUGAL & FLUXYS BELGIUM "D" \\
\hline ATTI-KAT & ESTORIL SOL"B" & IMMOBEL \\
\hline AXON HOLDINGS & FENALU LIMITED DATA & PICANOL \\
\hline BANK OF GREECE & IMMOBL.CON.GRAO-PARA & RETAIL ESTATES \\
\hline EDRASIS PSALLIDAS & LISGRAFICA & SABCA \\
\hline EKTER & LITHO FORMAS PORTUGUESA LIMITED DA & SIPEF \\
\hline ELTRAK PROPERTY & OREY ANTUNES & SOLVAC \\
\hline ELVIEMEK LAND DEVELOPMENT LOGIST.PK & SOCIETY AGUAS DA CURIA LIMITED DATA & SPADEL \\
\hline EMPORIKOS DESMOS & SONAE SGPS & TESSENDERLO \\
\hline FLEXOPACK & SONAGI LIMITED DATA & UMICORE \\
\hline FLR MLS C SARANTOPOULOS & TOYOTA CAETANO & VAN DE VELDE \\
\hline
\end{tabular}

Table A2 - List of Market Tier 2 stocks used in the portfolio optimization problem. 


\begin{tabular}{|c|c|c|}
\hline \multicolumn{3}{|c|}{ Market Tier 3} \\
\hline UK & Australia & Netherlands \\
\hline AVIVA & AGL ENERGY & AFC AJAX \\
\hline BRITISH AMERICAN TOBACCO & ALUMINA & AND INTL.PUBLISHERS \\
\hline BRITISH LAND & AUST.FNDTN.INV.COMPANY & ARCADIS \\
\hline BT GROUP & COCA-COLA AMATIL & ASML HOLDING \\
\hline IMPERIAL BRANDS & DEXUS PROPERTY GROUP & BATENBURG TECHNIEK \\
\hline KINGFISHER & FORTESCUE METALS GP. & BINCKBANK \\
\hline LLOYDS BANKING GROUP & GPT GROUP & BRILL \\
\hline PEARSON & JAMES HARDIE INDS.CDI. & EUROCOMMERCIAL \\
\hline PRUDENTIAL & NEWCREST MINING & HEINEKEN \\
\hline RECKITT BENCKISER GROUP & OIL SEARCH & HEINEKEN HLDG. \\
\hline ROLLS-ROYCE HOLDINGS & QBE INSURANCE GROUP & RELX \\
\hline SSE & SANTOS & ROYAL DUTCH SHELL A \\
\hline TESCO & STOCKLAND & STERN GROEP \\
\hline VODAFONE GROUP & TRANSURBAN GROUP & USG PEOPLE \\
\hline WPP & WESTFIELD & WOLTERS KLUWER \\
\hline
\end{tabular}

Table A3 - List of Market Tier 3 stocks used in the portfolio optimization problem.

\begin{tabular}{|c|c|c|}
\hline \multicolumn{3}{|c|}{ US Market } \\
\hline ADOBE SYSTEMS & CONOCOPHILLIPS & MERCK \& COMPANY \\
\hline AMAZON.COM & COSTCO WHOLESALE & MICROSOFT \\
\hline AMERICAN EXPRESS & DOW CHEMICAL & MORGAN STANLEY \\
\hline AMGEN & DUKE ENERGY & ORACLE \\
\hline APPLE & EXXON MOBIL & PEPSICO \\
\hline AT\&T & FEDEX & PFIZER \\
\hline BANK OF AMERICA & FORD MOTOR & PROCTER \& GAMBLE \\
\hline BANK OF NEW YORK MELLON & GENERAL ELECTRIC & REGENERON PHARMS. \\
\hline BIOGEN & GOLDMAN SACHS GP. & STARBUCKS \\
\hline BLACKROCK & HEWLETT-PACKARD & TARGET \\
\hline BOEING & HOME DEPOT & TEXAS INSTRUMENTS \\
\hline CATERPILLAR & INTEL. & TIME WARNER \\
\hline CHEVRON & INTERNATIONAL BUS.MCHS. & UNION PACIFIC \\
\hline CISCO SYSTEMS & JOHNSON \& JOHNSON & WAL MART STORES \\
\hline CITIGROUP & JP MORGAN CHASE \& CO. & WALT DISNEY \\
\hline COCA COLA & LOCKHEED MARTIN & \\
\hline COLGATE-PALM. & MCDONALDS & \\
\hline
\end{tabular}

Table A4 - List of U.S. Market stocks used in the portfolio optimization problem. 